\title{
Un varón con lupus miliar diseminado facial y granulomas faciales idiopáticos con evolución regresiva
}

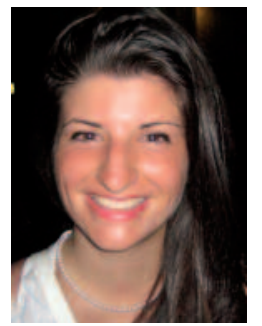

Diana Menis

Médico residente de Dermatología. Hospital Universitario 12 de Octubre. Madrid.

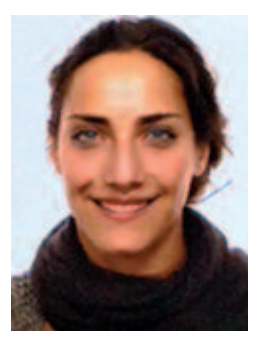

María Castellanos González Especialista en Dermatología. Hospital Universitario 12 de Octubre. Madrid.

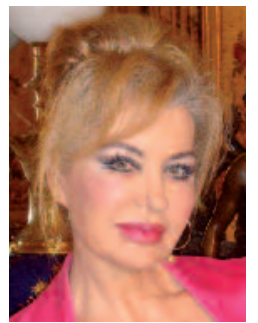

Aurora Guerra Tapia.

Profesora titular de Dermatología. Universidad Complutense de Madrid.

Jefa de la Sección de Dermatología. Hospital Universitario 12 de Octubre. Madrid.
Mi paciente es un varón de 14 años de edad que, desde hace 2 meses, presenta lesiones pruriginosas, que inicialmente estaban localizadas en mentón y frente y que han ido progresivamente incrementando su número hasta afectar a otras localizaciones de la cara, motivo por el que consulta. En la exploración física se aprecian numerosas pápulas milimétricas eritematosas y de bordes bien definidos, algunas aisladas y otras agrupadas en la región peribucal, surcos nasogenianos y párpados (figs. 1 y 2). No se objetivan comedones ni telangiectasias. El paciente niega sintomatología sistémica, antecedentes personales de enfermedades dermatológicas o la aplicación previa de cremas de corticoides o con fines cosméticos. Se decide realizar una biopsia de una de las lesiones, que muestra la presencia de un infiltrado inflamatorio linfocitario y granulomatoso, compuesto de histiocitos con abundantes células gigantes multinucleadas localizadas principalmente en áreas parafoliculares (fig. 3). La tinción con ácido peryódico de Schiff (PAS) y la tinción de Ziehl-Neelsen resultaron negativas. Se establece el juicio clínico de lupus miliar diseminado facial (LMDF). El resto de pruebas complementarias, que incluyen hemograma, velocidad de sedimentación globular, bioquímica, determinación de los niveles de calcio, test de Mantoux, radiografía de tórax y niveles de enzima convertidora de la angiotensina, fueron normales. Comenzamos tratamiento con doxiciclina $100 \mathrm{mg}$ cada 24 horas. En la primera revisión, al mes de tratamiento, las lesiones mejoraron notablemente, destacando el aplanamiento de las mismas y la disminución del picor.

El LMDF es una dermatosis inflamatoria de origen desconocido, con evolución autorresolutiva en un período de 1 a 4 años ${ }^{1,2}$, más frecuente y con una edad 


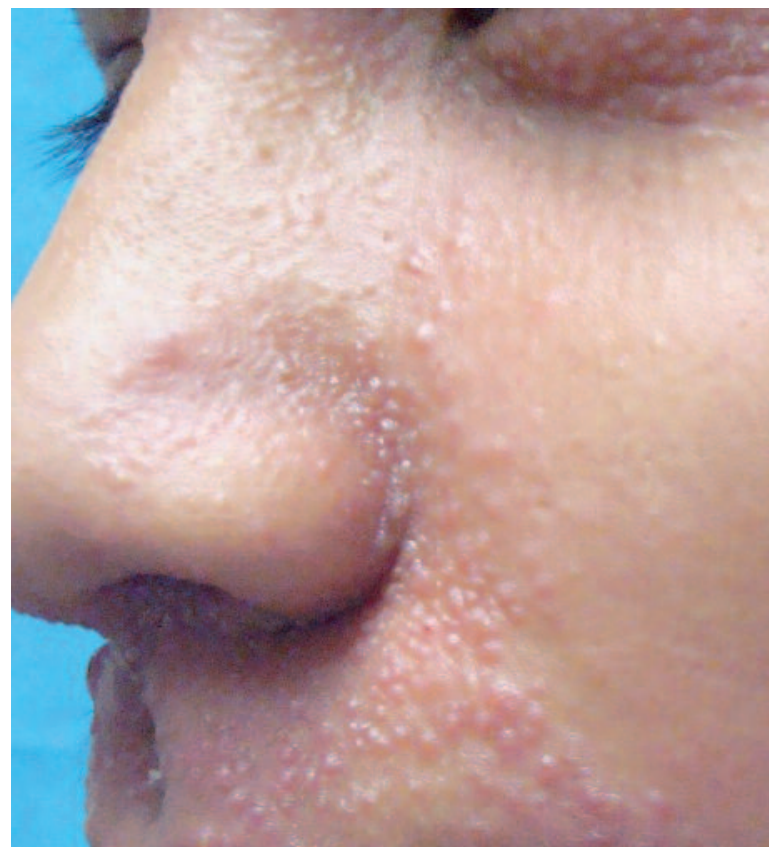

Figura 1. Detalle de las pápulas eritematosas en pliegue nasogeniano y párpado.

de aparición mucho más precoz (alrededor de los 20 años) en los varones. Clínicamente, se caracteriza por la aparición abrupta de pápulas eritemato-amarillentas de diámetro variable, entre 1 y $4 \mathrm{~mm}$, con localización centro-facial. Según un estudio sobre 35 pacientes reclutados entre 1989 y 2008 en el Departamento de Dermatología de la Universidad de Kitasato ${ }^{1}$, la localización periocular es la más frecuente $(91 \%)$ —en especial en el párpado inferior-, seguida por la perioral $(46 \%)$, las mejillas, incluyendo el surco nasogeniano $(40 \%)$, y la frente ( $35 \%$ de los casos). A menudo, las lesiones se extienden a mentón y cue$1 \mathrm{lo}^{3}$, y no es inusual la afectación de axilas, hombros, miembros superiores, manos y miembros inferiores, asociada a la presencia de lesiones en la cara. También hay casos descritos de localización exclusivamente extrafacial ${ }^{4}$. Mi paciente no presentaba lesiones fuera del área facial. Entre las hipótesis patogenéticas, bastante escasas, destaca la posibilidad de que se trate de una reacción granulomatosa secundaria a la liberación de

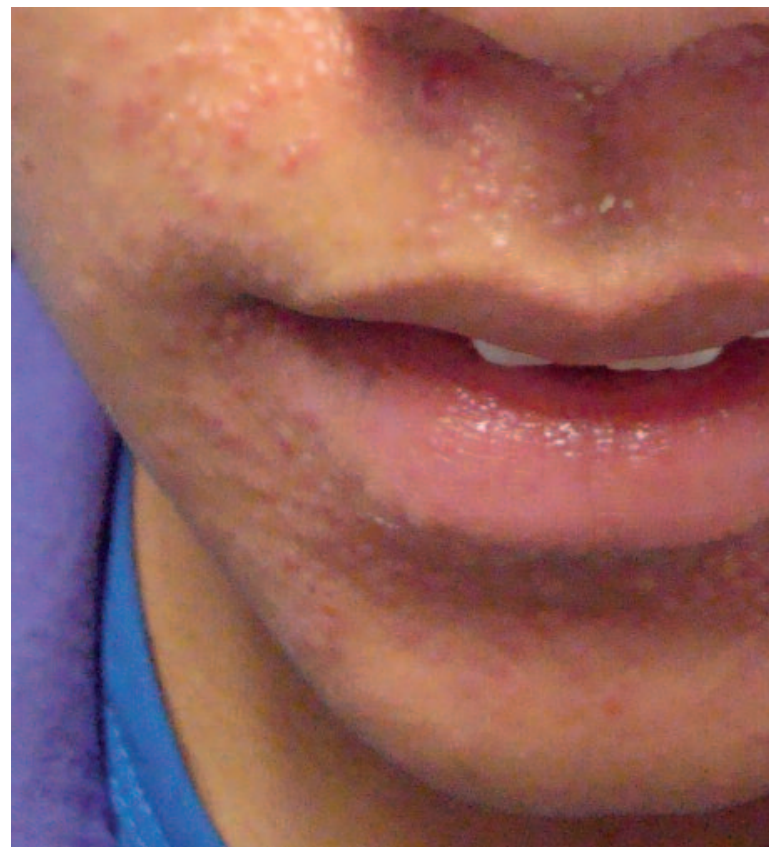

Figura 2. Pápulas eritematosas localizadas en mentón, triángulo nasogeniano y surco nasolabial.

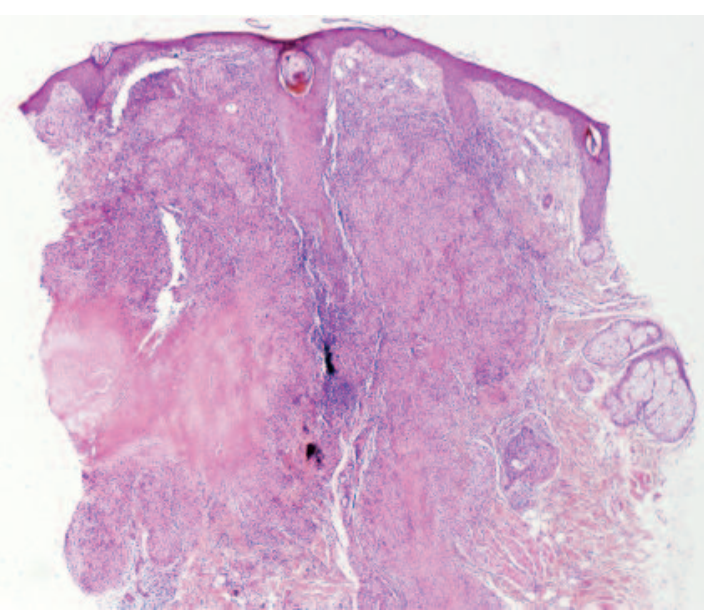

Figura 3. Estudio histopatológico. Infiltrado granulomatoso parafolicular compuesto principalmente por histiocitos, linfocitos y neutrófilos.

antígenos tras la ruptura de la pared del folículo piloso o de quistes epidérmicos. La histología varía según el estadio de las lesiones, de manera que en fase temprana se caracteriza por un infiltrado linfocitario con un pequeño porcentaje de neu- 


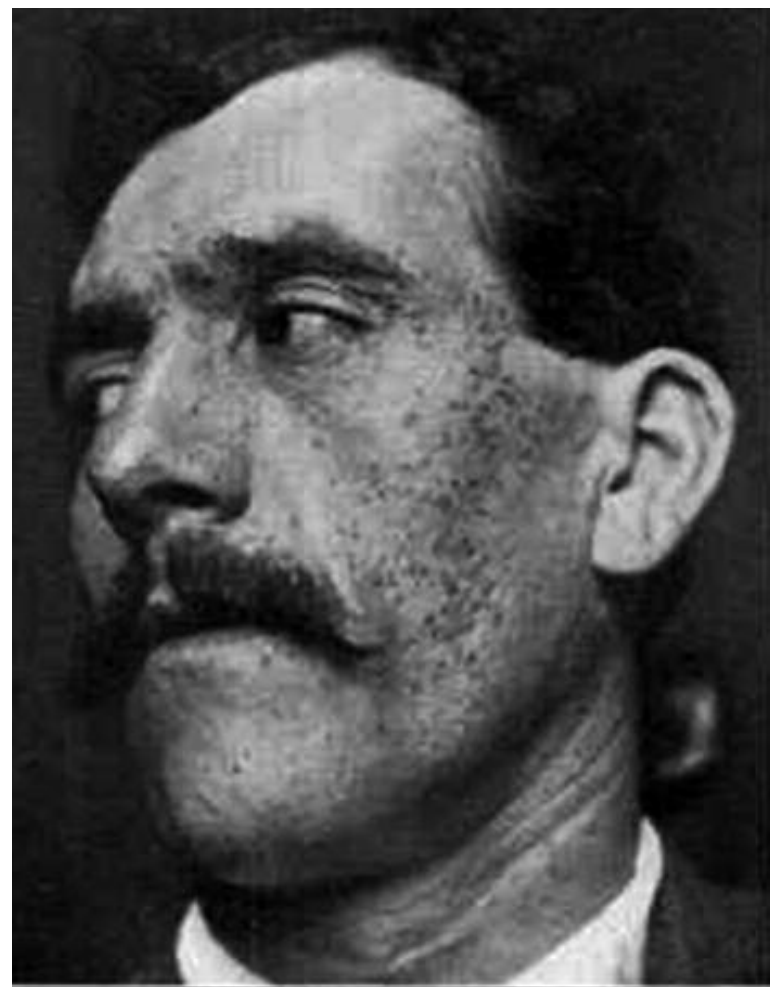

Figura 4. Caso de lupus miliar diseminado facial en los primeros años del siglo Xx. Tomada de: Radcliffe-Crocker H. editor. Disease of the skin. 3. ${ }^{\mathrm{a}}$ ed. Londres: Lewis; 1903.

trófilos, pasando por la formación de granulomas, inicialmente sin necrosis caseosa, pero que aparece en fase más tardía asociada a un aumento del infiltrado neutrófilo. Finalmente, se observa fibrosis perifolicular e infiltrado mixto histocitario y linfocitario.

El principal problema al que nos enfrentamos con esta entidad clínica es que no existen unos criterios diagnósticos bien establecidos, por lo que su identificación no siempre es fácil. Además, ha generado mucho debate entre diversos autores respecto a si se trata de una entidad independiente, o bien forma parte de un espectro de otras patologías que cursan con manifestaciones clínicas similares.

El LMDF fue mencionado en 1878 por Tibory Fox bajo el término de «lupus diseminado folicular» ${ }^{5}$, pero se describió por primera vez en 1903 como «lupus miliar diseminado facial» (fig. 4), y desde entonces ha sido relacionado con enfermedades como la tuberculosis, la sarcoidosis, el acné y la rosácea, creando términos como acné agminada, tuberculide rosaceiforme de Lewandowsky, acnitis de Barthélémy y el mismo LMDF. Esta variedad terminológica ha provocado que a menudo haya mucha confusión a la hora de definir esta entidad.

Hoy en día, la mayoría de los autores están de acuerdo en considerar esta enfermedad como una entidad independiente. Para recalcar este concepto, Skowron et al. introdujeron la utilización de un nuevo término: FIGURE (granulomas faciales idiopáticos con evolución regresiva $)^{6}$. Este acrónimo hace referencia a una condición caracterizada por la presencia de pápulas de localización centrofacial con predominio periorificial, sin asociación con sarcoidosis, acné o rosácea granulomatosa y que representa una reacción granulomatosa no específica a varios agentes tópicos y sistémicos. Algunos autores apoyaron este nuevo término, incluyendo este nuevo término al LMDF, la dermatitis granulomatosa periorificial de la infancia (CGPD) y la dermatitis perioral ${ }^{7}$. Según estos autores asiáticos, no existen diferencias clínicas o histológicas significativas entre el LMDF y la CGPD, por lo cual estas dos entidades se consideran parte del mismo proceso, que se presentan en diferente período vital. Por otra parte, en 2005 se propone englobar en la denominación común de «erupciones granulomatosas faciales idiopáticas» el LMDF, la rosácea granulomatosa, la CGPD y la sarcoidosis cutánea ${ }^{8}$. Según estos autores, esta denominación sería más apropiada, ya que el acrónimo FIGURE implica la evolución regresiva de las lesiones, mientras en estos trastornos el pronóstico resulta muy variable.

Por último, nos gustaría resaltar que no existe un tratamiento estándar de primera elección para esta patología. Hasta el día de hoy se han propuesto distintas alternativas con resultados variables, entre las que destacan los antibióticos, principalmente tetraciclinas. Otros tratamientos descritos son la dapsona, la isotretinoína, el tacrolimús, la clofa- 
zimina, el láser diodo de $1450 \mathrm{~nm}$ o el tranilast, entre otros, con resultados variables y a menudo decepcionantes ${ }^{2,9-11}$.

En conclusión, hemos presentado un caso de LMDF, trastorno con una historia de más de cien años, pero que hoy en día sigue generando discusión y dudas sobre su etiología y clasificación. Es un reto diagnóstico para el dermatólogo, y un inconveniente para el paciente, con importante repercusión en la calidad de vida, al ser una patología visible y desfigurante.

\section{BIBLIOGRAFÍA}

1. Amîruddîn D, Mii S, Fujimura T, Katsuoka K. Clinical evaluation of 35 cases of lupus miliaris disseminatus faciei. J Dermatol. 2011;38(6):618-20.

2. Naraf AL- Mutairi. Nosology and therapeutic options for lupus miliaris disseminatus faciei. J Dermatol. 2011;38:864-73.

3. Mullanax MG, Kierland R. Granulomatous rosacea. Arch Dermatol. 1970;101:206-11.
4. Kim DS, Lee KY, Shin JU, Roh MR, Lee MG. Lupus miliaris disseminatus faciei without facial involvent. Acta Derm Venereol. 2008;88(5):504-5.

5. Monsálvez V, Chico R, Ballen A, Postigo C. Dermatosis granulomatosas faciales. Actas Dermosifiliogr. 2010;101(7):6468.

6. Skowron F, Causeret AS, Pabion C, Viallard AM, Balme B, Thomas L. FIGURE: facial idiopathic granulomas with regressive evolución: is lupus miliaris disseminatus faciei still an acceptable diagnosis in the third millennium? Dermatology. 2000;201:287-9.

7. Misago N, Nakafusa J, Narisawa Y. Childhood granulomatous periorificial dermatitis: lupus miliaris disseminatus faciei in children? J Eur Acad Dermatol Venereol. 2004;19(4):470-3.

8. Makker R, Ramesh V. On the diagnosis of facial granulomatous dermatoses of obscure origin. Int J Dermatol. 2005;44: 606-9.

9. Kukano K, Tani M, Murata Y. Dapsone in the treatment of miliary lupus of de face. Br J Dermatol. 1983;109:57-62.

10. Daneshpazhooh M, Ehsani A, Toosi S, Robati RM. Isotretinoin in acne agminata. Saudi Med J. 2007;28(10):1600-2.

11. Guerra Tapia A, Rodríguez Peralto JL, González-Guerra E. Lesiones cutáneas asintomáticas diseminadas en cara (lupus miliar). Act Dermatol. 2003;5:407-9. 